\title{
Hericium erinaceus Mycelium Exerts Neuroprotective Effect in Parkinson's Disease-in vitro and in vivo Models
}

\author{
Pao-Pao Yang ${ }^{1,2}$, Chih-Yung Lin², Tzu-Yin Lin², and Win-Chin Chiang ${ }^{2, *}$ \\ ${ }^{1}$ Institute of Biotechnology and Pharmaceutical Research, National Health Research Institutes, Miaoli County, Taiwan, Republic of China \\ ${ }^{2}$ JOWIN BIOPHARMA Inc, Taiwan, Republic of China
}

*Corresponding author: Win-Chin Chiang, JOWIN BIOPHARMA Inc, Xizhi, New Taipei City, Taiwan, 9F-12, No. 97, Sec. 1, Xintai $5^{\text {th }}$ Road, Xizhi Dist, New Taipei City, Taiwan (22175), E-mail: winchiang@jowinbio.com

Received: 20 Feb, 2020 | Accepted: 13 Mar, 2020 | Published: 20 Mar, 2020

Citation: Yang PP, Lin CY, Lin TY, Chiang WC (2020) Hericium erinaceus Mycelium Exerts Neuroprotective Effect in Parkinson's Disease-in vitro and in vivo Models. J Drug Res Dev 6(1): dx.doi.org/10.16966/2470-1009.150

Copyright: (C) 2020 Yang PP, et al. This is an open-access article distributed under the terms of the Creative Commons Attribution License, which permits unrestricted use, distribution, and reproduction in any medium, provided the original author and source are credited.

\begin{abstract}
Hericium erinaceus (H.E.) is a well-known edible and folk medicinal fungi in Japan, China and other Asian countries without harmful effects. It has been recognized that this unique mushroom is capable of keeping the brain healthy, supporting the immune system to help prevent gastric cancer and other diseases, boosting mood and concentration, decreasing inflammatory processes in the body. But more scientific researchers are needed to confirm its nutritional and medicinal effects. In the present study, we investigated the effects of Hericium erinaceus mycelium (H.E. mycelium) against 1-methyl-4-phenylpyridinium(MPP+)-induced neurotoxicity in PC12 cells and 1-methyl-4-phenyl-1,2,3,6-tetrahydropyridine (MPTP)-induced Parkinsonian mice. In the cell viability results, treatment with H.E.mycelium increased the cell viability in MPP+-treated cells and induced antioxidant activity in PC12 cells. H.E. mycelium also reduced MPTP-induced loss of dopamine concentration level and tyrosine hydroxylase (TH) positive cells in mice. Our results suggest that H.E.mycelium performs significant protection of dopaminergic neuron under severe conditions and is very effective in the treatment of damaged neuron in the brain to recover in the case of Parkinson's disease.
\end{abstract}

Practical applications: In this article, we provide science-based evidence related to H.E.mycelium to be a potential effective material for the treatment and prevention of Parkinson's disease.

Keywords: Hericium erinaceus mycelium; Dopamine; Neuroprotective; Parkinson's disease; Tyrosine hydroxylase

\section{Introduction}

Parkinson's disease (PD) is one of the most common progressive neurodegenerative disease that is characterized by the loss of dopaminergic neurons in the Substantia Nigra par compacta (SNpc) region of the brain [1], which results in motor problems including bradykinesia, akinesia, muscular rigidity, resting tremor, and postural instability [2]. In the disease model of PD, the involvement of the drug 1-methyl-4-phenyl-1, 2, 3, 6-tetrahydropyridine (MPTP), is most widely used among animal models of Parkinson's disease [3]. The MPTP is metabolized into the toxic cation 1-methyl-4phenylpyridinium ion (MPP+) by the enzyme monoamine oxidase $\mathrm{B}$, which causes the neurotoxic effect to impair the dopaminergic nigrostriatal neurons [4].

Hericium erinaceus (H.E.) a well reputed edible mushroom, also known as monkey head mushroom in Chinese, Yamabushitake in Japanese or Lion's mane mushroom in English, has been widely reported to use as food and folk medicine in Japan, [5] China and other Asian countries without harmful effects [6]. Several evidences demonstrated that it possesses a wide range of benefits, such as anticancer [7,8], antimicrobial [9], antioxidant [10], anti aging [11], anti-hyperglycemic [12], anti-hyperlipidemic activity [13], gastroprotective [14], immunomodulating and neuroprotective activity (Alzheimer's Disease and Parkinson's Disease) [15-17], protection of neuropathic pain [18], depressive symptoms [19] and presbycusis [20].

As the mycelium is inoculated in grain spawn, lion's mane mushroom grows in large snowball-like formations, which is called the fruiting body. Hericenones, the benzyl alcohol derivatives with simple fatty acids, only exist in the fruiting body. A group of erinacines (erinacines A-K and P-S) which are diterpenoid derivatives have been identified only from the mycelium, and erinacine $\mathrm{A}$ is most rich in the mycelium [21]. It was demonstrated that eight of erinacines (A-I) could enhance nerve growth factor (NGF) release [21] and the erinacenes are more potent inducers of NGF synthesis than hericenones. They not only have an enhancing effect on NGF synthesis in astroglial cells in vitro but also can increase both NGF and catecholamine content in the hippocampus of rats [11]. The increased amount of NGF, in turn, enhances neuronal survival in different brain regions and substantially improves animal behavioral activity. 
The aim of this present study was to explore the neuro protective effects of Hericium erinaceus mycelium (H.E. mycelium) using MPP+-treated PC12 cells or MPTP-induced PD mouse model that is associated with protection against loss of the neurotransmitter or dopaminergic neuron in vitro and in vivo.

\section{Materials and Methods}

\section{Preparation of Hericium erinaceus mycelium (H.E. mycelium)}

H.E. mycelium powder (mesh size\#100, RH6408) were obtained from FUNGUS BIOTECH, Co. Ltd. Yilan, Taiwan, where toxin-free and pesticide-free of Hericium erinaceus solid state fermentation was exercised and the mycelium was collected afterward and dried to the moisture content of less than $7 \%$. The H.E. mycelium yellowish powder was then further grinded into smaller particles through a spiral jet mill (OM2 micronizer, Sturtevant Inc. Hanover, MA USA) to induce the cell wall-broken effect with a particle size distribution of $\mathrm{D} 75<50 \mu \mathrm{m}$ at FORMOSAN NANO BIOLOGY Co. Ltd, Taichung, Taiwan. The cell wall-breaking technology greatly contributed to the increased release rate of active ingredients from the fine H.E. Mycelium particle powder.

\section{Cell viability}

The MTS assay is a colorimetric method and usually used to assess cell proliferation, cell viability and cytotoxicity. Its protocol is based on the reduction of the MTS tetrazolium compound by viable cells to generate a colored formazan dye that is soluble in cell culture media. PC12 cell is a cell line derived from a phenochromocytoma of the rat adrenal medulla and was used in this study. The PC12cells were maintained at $3^{\star} 10^{4}$ cells /well in 96 wells plate with $100 \mu \mathrm{L}$ of DMEM at $37^{\circ} \mathrm{C}$ in an incubator containing $5 \%$ carbon dioxide for $24 \mathrm{~h}$. Briefly, after cells had attached, cells were treated with MPP+ for $72 \mathrm{~h}$ in the presence of ethanol extracts of test samples which was in $0.4 \%$ DMSO (Dimethyl Sulfoxide) solution. Afterward, MTS solution was added to each cell well and made it into a colored solution. The whole process was performed triplicate. The absorbance of the colored solution in each cell was measured at $570 \mathrm{~nm}$ using a microplate reader. To assess the neuroprotective effects of H.E. mycelium on the PC12 cells with MPP+-induced toxicity, the cells were treated with different concentration of H.E. mycelium at 8, 40,200, $1000 \mu \mathrm{g} / \mathrm{mL}$, respectively, and different concentrations of Ganoderma lucidum (Reishi) at 8, 40, $200,1000 \mu \mathrm{g} / \mathrm{mL}$, respectively, with addition of $10 \mathrm{mM} \mathrm{MPP}+$ solution to reach the final concentration of $1 \mathrm{mM} \mathrm{MPP}+$ in the cell. Cell viability was assessed $72 \mathrm{~h}$ later by measuring the absorbance of the colored solution. The survival rate of the control group was normalized as the basis for that of the other groups to compare and calculate.

\section{DPPH scavenging assay}

The free radical scavenging activity of the tested extract was performed by using 2,2-Diphenyl-1-picrylhydrazyl (DPPH) which was at $0.1 \mathrm{mM}$ Methanol solution. The extract fractions at different concentrations were prepared with distilled water/ethanol (50:50). The antioxidant standard compound, ascorbic acid, at $1 \mathrm{mg} / \mathrm{mL}$ was used as positive standard for the comparison purpose. A fresh stock solution of the standard compound was prepared before each analysis. The absorbance changes in color from deep purple to light yellow at $517 \mathrm{~nm}$ were measured by using a spectrophotometer after $30 \mathrm{~min}$ of reaction. The solution of DPPH at $0.1 \mathrm{mM}$ was used as a control.

To express the radical scavenging activity, the $\mathrm{IC}_{50}$ parameter was employed and it is defined as the concentration of substrate that brings about $50 \%$ loss of the DPPH free radical. The $\mathrm{IC}_{50}$ value of H.E. mycelium is the concentration of H.E. mycelium required to inhibit $50 \%$ of the DPPH free radical. Likewise, the $\mathrm{IC}_{50}$ value of Ganoderma lucidum was so defined. The percent inhibition of DPPH free radical by a test sample calculated by using the following formula:

$\%$ of inhibition $=[(\mathrm{dc}-\mathrm{dt}) / \mathrm{dc}] \times 100$

Where $\mathrm{dc}$ was the absorbance of control reaction and $\mathrm{dt}$ was the absorbance in presence of test or standard sample.

\section{Animals and treatment}

Adult male C57BL/6 Narl mice weighing 20-30 g (8-12 weeks old) were purchased from the National Laboratory Animal Center (Taipei, Taiwan) and used in this study. Mice were kept at constant room temperature $\left(20-22^{\circ} \mathrm{C}\right)$ and humidity $(50 \%-70 \%)$ with a $12 \mathrm{~h} \mathrm{light/}$ dark cycle (7:00-19:00) in the Animal Centers of the National YangMing University, Taiwan. Standard diet and water were available Ad libitum during the experiment. The care of animals was carried out in accordance with institutional and international standards (Principles of Laboratory Animal Care, NIH); and all experiments were approved by the Institutional Animal Care and Use Committees of the National Yang-Ming University, Taiwan (IACUC No. 1050306). All studies involving animals were conducted in accordance with the ARRIVE guidelines [22,23].

Five groups (fifteen mice in each group) were randomly assigned to a control group (saline, i.p.) plus $\mathrm{H}_{2} \mathrm{O}$ (p.o.), MPTP group $(20 \mathrm{mg}$ / kg, i.p; Tokyo chemical Industry, TCI) plus $\mathrm{H}_{2} \mathrm{O}$ (p.o.) and MPTP $(20$ $\mathrm{mg} / \mathrm{kg}$, i.p.) plus H.E. mycelium groups $(0.1 \mathrm{~g} / \mathrm{kg}, 0.3 \mathrm{~g} / \mathrm{kg}$ and $1 \mathrm{~g} / \mathrm{kg}$., p.o.). Mice were received intraperitoneal injection of saline or MPTP once a day in the beginning 5 consecutive days and oral administration of $\mathrm{H}_{2} \mathrm{O}$ or H.E. mycelium in the duration of 30 -day period. The control group of animal received an equivalent volume of saline. The animals were sacrificed 30 days after the last treatment of five groups and then brains were dissected to the left and right cerebrum. The right cerebrum was removed to determine immunohistochemistry for tyrosine hydroxylase and the left cerebrum of striatum was rapidly removed to determine concentration of dopamine neurotransmitter.

\section{Determination of the concentration of dopamine neurotransmitter}

The mice were sacrificed under pentobarbital after completion of the treatment. The left cerebrum of striatum was quickly removed and homogenized in a stock solution containing $0.1 \mathrm{M} \mathrm{HClO}_{4}, 0.1 \mathrm{mM}$ EDTA, $0.1 \mathrm{mM} \mathrm{Na} \mathrm{S}_{2} \mathrm{O}_{5}$ and centrifuged at $13,000 \mathrm{rpm}$ for $10 \mathrm{~min}$ at $4^{\circ} \mathrm{C}$. The supernatant was harvested, filtered with $0.45 \mu \mathrm{m}$ poresize filters, and reserved at $-80^{\circ} \mathrm{C}$ until analysis. The concentration of dopamine was determined by using High-Performance Liquid Chromatography (HPLC) with electrochemical detection.

\section{High-performance liquid chromatography assay for} neurotransmitter

HPLC electrochemical detection was performed to quantify the concentration of dopamine. The HPLC system consisted of a pump (BAS PM-92E; Bioanalytical Systems, West Lafayette, IN, USA), a refrigerated microsampler $(\mathrm{CMA} / 200)$ and a sample injector (CMA/240) with a $20 \mu \mathrm{L}$ loop (CMA, Stockholm, Sweden), and a digital amperometric electrochemical detector (Decade II; Antec Leyden BV, Zoeterwoude, The Netherlands). For the determination of the dopamine concentration, $15 \mu \mathrm{L}$ samples were injected into the HPLC system. The mobile phase consisted of $0.74 \mathrm{mM}$ sodium1-Octanesulfaoate (SOS), $100 \mathrm{mM}$ phosphate sodium salt, $0.027 \mathrm{mM}$ EDTA, $2 \mathrm{mMKCl}, 125 \mathrm{~mL}$ methanol, which was delivered at a flow 
rate of $500 \mu \mathrm{L} / \mathrm{min}$. A reversed-phase $\mathrm{C}-18$ column $(100 \times 4.6 \mathrm{~mm}$, $2.6 \mu \mathrm{m})$ was used for sample separation. The applied potential of the glassy carbon electrode was $650 \mathrm{mV}$ to the reference electrode $(\mathrm{Ag} / \mathrm{AgCl})$, and the range setting was $5 \mathrm{nA}$ for the determination of neurotransmitters. The data acquisition and analysis were performed using EZChrom software (Scientific Software, San Ramon, CA, USA).

\section{Immunohistochemistry (IHC) for Tyrosine Hydroxylase (TH) in mouse brain}

For measuring tyrosine hydroxylase immunohistochemistry, the mice were anesthetized with pentobarbital after completion of the treatment. The right cerebrum was removed and soaked at $4^{\circ} \mathrm{C}$ in the paraformaldehyde (PFA) alone, and maintained in $30 \%$ sucrose at $4^{\circ} \mathrm{C}$ until they sank. The cerebrum was sectioned at $10 \mu \mathrm{m}$. All sections were stained for tyrosine hydroxylase measurements.

\section{Data analysis and statistical assessment}

Data were expressed as the mean \pm SEM. Analysis of variance was used to access the statistical significance for repeated measures of the data, and the differences among the individual mean values in different groups were analyzed by ANOVA followed by the Newman-Keuls test. The differences were considered to be significant at $\mathrm{p}<0.05$.

\section{Results}

\section{DPPH scavenging activity (antioxidant activity)}

The DPPH radical scavenging assay was determined spectrophotometrically. The radical scavenging capacities of the tested extract were performed by DPPH assay and results were shown in figure 1. DPPH assay was one of the most widely and commonly used methods for screening antioxidant activity of plant or mushroom extracts. In the figure 1, DPPH scavenging activities of H.E. mycelium, and reference antioxidants capacities of Ganoderma lucidum (Reishi) obtained from FUNGUS BIOTECH, Co. Ltd., Yilan, Taiwan and ascorbic acid were analyzed in this study. The concentrations of H.E. mycelium at the range of 62.5 and $1000 \mu \mathrm{g} / \mathrm{mL}$ exhibited positive DPPH scavenging activities in a concentration-dependent manner as had shown in figure 1. The higher the concentration of H.E. mycelium at, the more the DPPH radical scavenging activity. As compared, Ganoderma lucidum showed a relatively lower radical scavenging effect than H.E. mycelium. The radical scavenging effect of the H.E. mycelium can also be observed by comparing $\mathrm{IC}_{50}$ value. A lower $\mathrm{IC}_{50}$ value indicates a higher antioxidant activity. The $\mathrm{IC}_{50}$ value of the H.E.

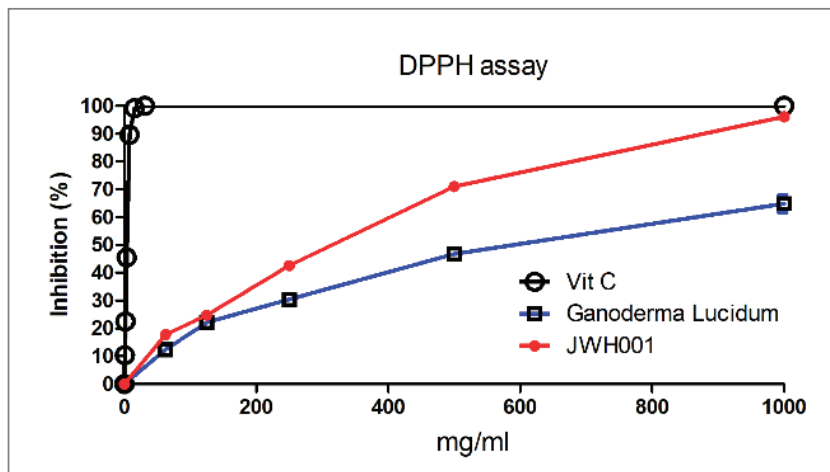

Figure 1: DPPH (1-Diphenyl-2-picrylhydrazyl) scavenging effect of H.E. mycelium and Ganoderma lucidum. Ascorbic acid (Vitamin C) was used as positive control. Data are presented as means \pm SEM. mycelium was $217.2 \mu \mathrm{g} / \mathrm{mL}$, compared to that of Ganoderma lucidum with $550.4 \mu \mathrm{g} / \mathrm{mL}$. It indicated H.E. mycelium had a better radical scavenging effect than Ganoderma lucidum. Overall, these findings support that H.E. mycelium exerted a good antioxidant activity in PC12 cells.

\section{H.E. mycelium protected MPP+- induced neurotoxicity in PC12 cell}

The protective ability of H.E. mycelium from the cytotoxicity of MPP+ in PC12 cells was measured by using the MTS assay. The results of the measurement, as shown in figure 2, revealed the decrease of cell viability of PC12 cells after exposure to $1 \mathrm{mM} \mathrm{MPP}+$ for $72 \mathrm{~h}$. In the presence of $1 \mathrm{mM} \mathrm{MPP}+$, H.E. mycelium at the concentration range of 40,200 and $1000 \mu \mathrm{g} / \mathrm{mL}$ showed a statistically significant protective

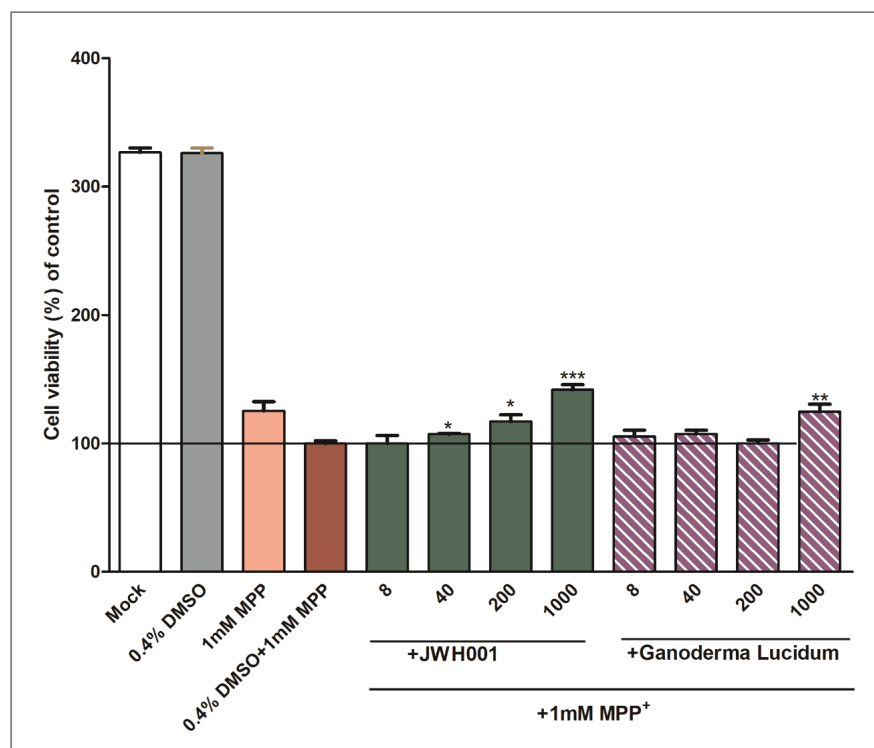

Figure 2: H.E. mycelium protected MPP+ induced neurotoxicity in PC12 cells. PC12 cells were treated with H.E. mycelium or Ganoderma lucidum with MPP+. Cell viability was measured by MTS assay. Data are presented as means \pm SEM. One way ANOVA and Newman-Keuls tests were used to analyze the data. ${ }^{*} P<0.05 ;{ }^{* *} p<0.01 ;{ }^{* * *} p<0.001$ compared to $0.4 \%$ DMSO with $1 \mathrm{mM} \mathrm{MPP}+$.

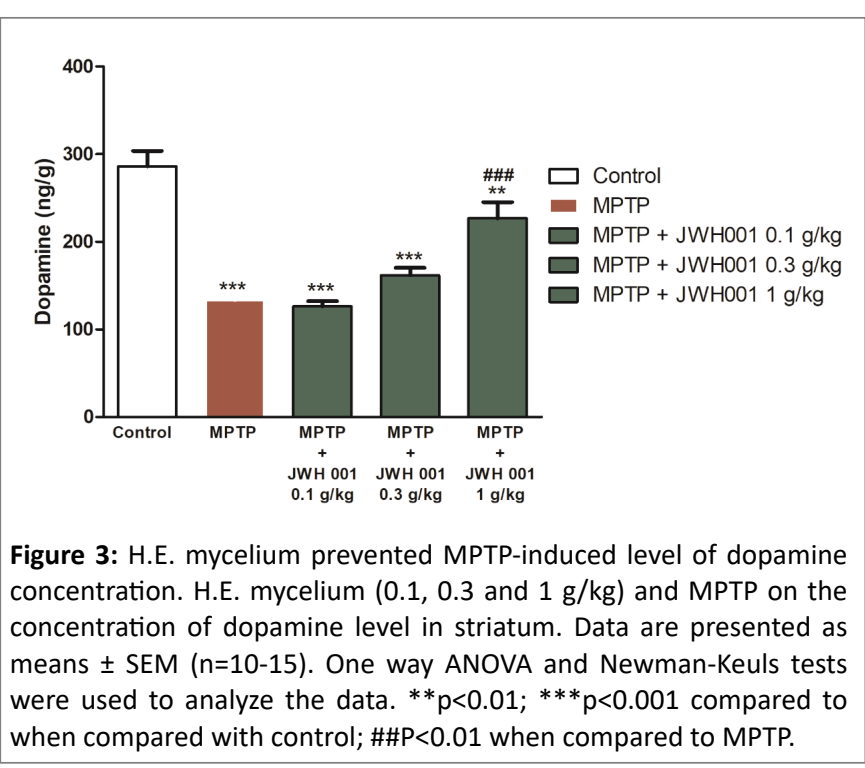


effect in a concentration dependent manner. A high concentration exerted a better neuroprotective effect. But, Ganoderma lucidum showed a significant protective effect in the PC12 cells only at high concentration of $1000 \mu \mathrm{g} / \mathrm{mL}$, as shown in figure 2 . It can be concluded that H.E. mycelium exerted a very good protective effect against the $\mathrm{MPP}+$ neurotoxicity in PC 12 cells.

\section{H.E. mycelium prevented MPTP-induced reduction of dopaminergic neuron}

The protective effect of H.E. mycelium against MPTP-induced toxic damage on dopaminergic neuron can be interpreted by looking at the reduction of decreased dopamine level in the striatum of the brain after H.E. mycelium treatment. The dopamine concentration was measured by using HPLC. From figure 3, one can recognize that five injections of MPTP $(20 \mathrm{mg} / \mathrm{kg}$, i.p.) reduced to about $46 \%$ of dopamine level of striatum in mice, compared to the control group. As expected, the oral administration of H.E. mycelium at 0.3 and $1 \mathrm{~g} / \mathrm{kg}$ increased the dopamine level to $56 \%$ and $79 \%$, respectively, which indicated that the protective effect of H.E. mycelium was $20 \%$ and $70 \%$ at 0.3 and $1.0 \mathrm{~g} /$ $\mathrm{kg}$, respectively, as compared to the MPTP group [24]. These results demonstrated that H.E. mycelium exerted the protective effect against the MPTP-induced dopamine neuron damage.

\section{H.E. mycelium prevented MPTP-induced death of tyrosine hydroxylase(TH)-positive neuron in striatum in mice}

The enzyme tyrosine hydroxylase $(\mathrm{TH})$ converts the amino acid L-tyrosine into 3,4-dihydroxyphenylalanine (L-DOPA) which then converts to dopamine by decarboxylation and then norepinephrine and epinephrine are produced in series in the pathway in both central and peripheral nervous systems [25]. The biosynthetic of dopamine pathway is illustrated in figure 4 . Therefore, the activity of tyrosine hydroxylase can influence the dopamine level of striatum in brain tissue and the reduction of tyrosine hydroxylase activity represents the damage of dopaminergic neurons in the brain. Figure 5 shows a large number of TH-positive cells in the control group and MPTP caused $63 \%$ reduction in TH-positive cells in mice compared with the control group. After treatment of H.E. mycelium at $0.1,0.3$ and
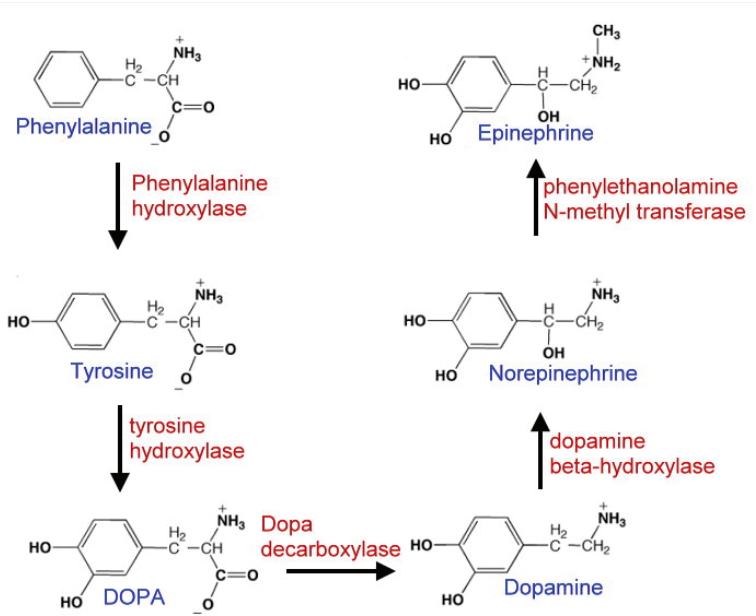

Figure 4: The biosynthetic pathway of dopamine neurotransmitters. Tyrosine hydroxylase is the rate limiting enzyme of the pathway. Phenylalanine hydroxylase converts phenylalanine to tyrosine, tyrosine hydroxylase hydroxylates tyrosine to L-DOPA. DOPA is converted to dopamine by decarboxylase. Dopamine converts to Norepinephrine by dopamine- $\beta$-hydroxylase and norepinephrine to epinephrine by phenylethanolamine $\mathrm{N}$ - methyl transferase.

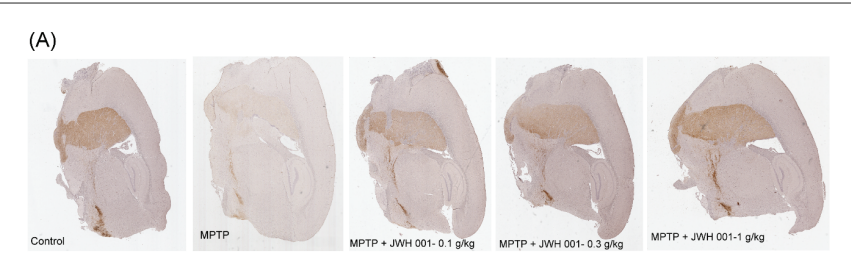

(B)

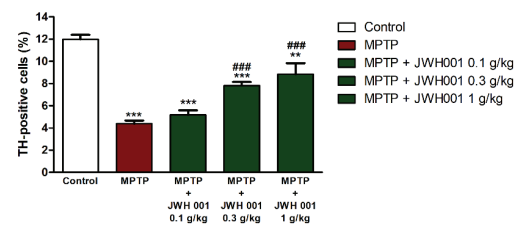

Figure 5: H.E. mycelium prevented MPTP-induced death of TH (Tyrosine Hydroxylase)-positive neuron in striatum in mice.

(A). TH expression in striatum was assessed by immunohistochemistry. (B). Quantitative analysis of TH-positive cells in the striatum of mouse brain. Dopamine neurons from mice are resistant to MPTP neurotoxicity. There is significant difference in the average number of TH positive neurons between the striatum of $0.3,1 \mathrm{~g} / \mathrm{kg}$ and that of MPTP group. Data are presented as means \pm SEM $(n=10-15)$. One way ANOVA and Newman-Keuls tests were used to analyze the data. $* * \mathrm{P}<0.01 ; * * * \mathrm{p}<0.001$ when compared to control; \#\#\#P<0.001 when compared to MPTP.

$1 \mathrm{~g} / \mathrm{kg}$ in the MPTP-induced mice, it improved the TH density in the substantia nigra area of the brain by $18 \%, 78 \%$ and $100 \%$ at these three concentrations respectively, compared with the MPTP damage group. These findings confirmed that H.E. mycelium exerted protection from MPTP-induced death of TH-positive neurons in corpus striatum of mice.

\section{Discussion}

In this present study we demonstrates that the neuroprotective effect of H.E. mycelium against MPP+-induced toxicity in neuronal PC12 cell lines and MPTP-induced striatal dopamine neuron damage in mice model. Our data strongly suggests that H.E. mycelium intake could be a potential treatment of PD (Parkinson's disease).

Parkinson's disease (PD) is characterized by a progressive degeneration of dopaminergic neurons in the substantia nigra pars compacta $(\mathrm{SNpc})$ region of the brain [1], and N-methyl-4-phenyl1,2,3,6-tetrahydropyridine (MPTP) has been widely used to induce PD in the animal model. MPTP induces PD through the death of dopaminergic cell by its active metabolite, 1-methyl-4-phenyl-2,3,dihydropyridinium (MPP+) [3]. By using $\mathrm{MPP}+$ in PC12 cells, as a cellular model of PD, we have elucidated the role of H.E. mycelium in modulating and reducing the loss of dopaminergic neurons. In the PC12 cell viability experiment, H.E. mycelium exhibited positive protective effect to dopamine neuron in mice which represents the similar neuroprotective properties in dopamine neurons to human PD [26,27]. Numerous studies have shown that MPP+-induces neurotoxicity in cell and zebrafish [28], and induces loss of dopaminergic neurons [2931]. Administration of MPP+ $(1 \mathrm{mM})$ induced cell death in PC12 cells is in consistent with several previous studies. MPP+-induced loss of dopaminergic neurons indicates the selective damage to dopamine neurons in cells. $H$. erinaceus extract promoted NGF synthesis by hericenones from fruit bodies (Hericenone $\mathrm{C}-\mathrm{H}$ ) and by erinacines from mycelium (erinacines A-I) [15]. H.E. mycelium reduced MPP+induced PC12 cell toxicity in a concentration dependent manner, indicating that H.E. mycelium could contribute to the protective effect 
of dopaminergic neuron in PC12 cells. In 2002, Park YS, et al. [32] reported that H. E. enhanced the synthesis of NGF (Nerve Growth Factor) and BDNF (Brain-Derived Nerve Factor) in PC12 cells. BDNF and NGF can be strongly expressed by dopaminergic neurons in SNpc. But, in human and animal models of Parkinson's disease, the expression of NGF and BDNF levels are decreased $[33,34]$. Thus, NGF level corresponds very well to the severity of PD. By summing up all the evidence, a theory can be formed, in which erinacines of H.E. mycelium upon ingested are transported to the brain where significant amount of NGF molecules are formed. The NGF molecules then promote brain nerves in substantia nigra to grow where dopaminergic neuron produces dopamine to a level that alleviates the Parkinson's disease.

In terms of effective dosage of H.E. mycelium, it was shown in this study that it performed much better than Ganoderma lucidum. Although it has been reported that Ganoderma lucidum has abilities to induce neuronal differentiation and prevent NGF-dependent PC12 neuronal cells from apoptosis [35], H.E. mycelium was more effective in neuroprotection than Ganoderma lucidum in this PC12 cells study. It may be due to that fact that a new cell-wall breaking technology was employed and also finer particles were produced. It improves the release of active ingredients from smaller H.E. mycelium particle powders.

By using DPPH radical scavenging assay, widely used method to evaluate the ability of mushroom to scavenge free radicals generated from DPPH reagent [36,37], the present data also showed that H.E. mycelium had more effective in scavenging DPPH radicals when compared to Ganoderma lucidum. The radical inhibition effect of Ganoderma lucidum was $59.3 \%$ at concentration of $1000 \mu \mathrm{g} / \mathrm{ml}$, when compared with H. E. mycelium's $96.1 \%$ and with ascorbic acid's $100 \%$ at the same concentration.

Administration of MPTP was known to decrease the activity of neuron and the density of TH-positive neurons, indicating that the degeneration of the dopaminergic neurons in $\mathrm{SNpc}[38,39]$. In our present study, injection of MPTP into C57BL/6 Narl mice induced significant reduction of dopamine level and $\mathrm{TH}$-positive area in the striatum, suggesting that MPTP initially affects the dopaminergic cell body in the substantia nigra pars compacta $(\mathrm{SNpc})$ and then the striatum where dopaminergic cells exert it's function of release of dopamine. Tyrosine hydroxylase $(\mathrm{TH})$ is the initial enzyme in the catecholamine synthesis pathway [40] and dopamine biosynthesis in the central nervous system [41]. TH is activated to form more DOPA and then dopamine by decarboxylation which is transferred into the synaptic vesicle by the vesicular monoamine transporter (VMAT). In addition, the loss of $\mathrm{TH}$ activity or expression is thought to contribute to dopamine deficiency, which is the most prominent at media level of SNpc (substantia nigra pars compacta) [39]. In our current study, the level of dopamine concentration and immunohistochemistry for $\mathrm{TH}$ positive neurons revealed that the loss of dopamine neuron in PD mice was dramatically prevented after treatment of H.E. mycelium.

In consistent with other research, the results of our present study showed that the immuno reactivity of $\mathrm{TH}$ was significantly decreased in MPTP-treated mice, suggesting that the majority of dopaminergic neurons were lost in the Parkinson's disease mouse model [42]. The death of dopaminergic neurons led to decrease dopamine level in the substantia nigra. The expression of $\mathrm{TH}$ positive neuron and concentration of dopamine level were increased with the concentration of H.E. mycelium from $0.3 \mathrm{~g} / \mathrm{kg}$ to $1 \mathrm{~g} / \mathrm{kg}$ in MPTP-induced mice. This evidence suggested that the neuroprotection and anti-oxidation is involved in the protective effect of H.E. mycelium on dopaminergic neurons.

\section{Conclusion}

In conclusion, we have used cell culture and animal models to demonstrate the neuroprotective effect of H.E. myceliumin the PD model. Our results indicate that H.E. mycelium increased the cell viability of the MPP+ treated cells and performed in a very effective antioxidative effect in vitro cell culture model using PC 12 cells. In vivo, H.E. mycelium exerts significant neuroprotective effect by increasing the dopamine level and the activity of tyrosine hydroxylase $(\mathrm{TH})$ in mice striatum. To sum up, our results suggest that H.E. mycelium performs significant protection of dopaminergic neuron under severe conditions and is very effective in the treatment of damaged neuron in the brain to recover in the case of Parkinson's disease.

\section{References}

1. Rane P, Sarmah D, Bhute S, Kaur H, Goswami A, et al. (2018) Novel Targets for Parkinson's Disease: Addressing Different Therapeutic Paradigms and Conundrums. ACS Chem Neurosci 10: 44-57.

2. Barbeau A (1968) Dopamine and dopamine metabolites in Parkinson's disease-a review. Proc Aust Assoc Neurol 5: 95-100.

3. D'Amato RJ, Lipman ZP, Snyder SH (1986) Selectivity of the parkinsonian neurotoxin MPTP: toxic metabolite MPP+ binds to neuromelanin. Science 231: 987-989.

4. Holtz WA, O'Malley KL (2003) Parkinsonian mimetics induce aspects of unfolded protein response in death of dopaminergic neurons. J Biol Chem 278: 19367-19377.

5. Wang M, Gao Y, Xu D, Konishi T, Gao Q (2014) Hericium erinaceus (Yamabushitake): a unique resource for developing functional foods and medicines. Food Funct 5: 3055-3064.

6. Ulziijargal E, Mau JL (2011) Nutrient compositions of culinarymedicinal mushroom fruiting bodies and mycelia. Int J Med Mushrooms 13: 343-349.

7. Lee JS, Hong EK (2010) Hericium erinaceus enhances doxorubicininduced apoptosis in human hepatocellular carcinoma cells. Cancer Lett 297: 144-154.

8. Li G, Yu K, Li F, Xu K, Li J, et al. (2014) Anticancer potential of Hericium erinaceus extracts against human gastrointestinal cancers. J Ethnopharmacol 153: 521-530.

9. Yim MH, Shin JW, Son JY, Oh SM, Han SH, et al. (2007) Soluble components of Hericium erinaceus induce NK cell activation via production of interleukin-12 in mice splenocytes. Acta Pharmacol Sin 28: 901-907.

10. Malinowska E, Krzyczkowski W, Lapienis G, Herold F (2009) Improved simultaneous production of mycelial biomass and polysaccharides by submerged culture of Hericium erinaceum: optimization using a central composite rotatable design (CCRD). J Ind Microbiol Biotechnol 36: 1513-1527.

11. Li IC, Chen YL, Chen WP, Lee LY, Tsai YT, et al. (2014) Genotoxicity profile of erinacine A-enriched Hericium erinaceus mycelium. Toxicol Rep 1: 1195-1201.

12. Liang B, Guo Z, Xie F, Zhao A (2013) Antihyperglycemic and antihyperlipidemic activities of aqueous extract of Hericium erinaceus in experimental diabetic rats. BMC Complement Altern Med 13: 253.

13. Yang BK, Park JB, Song CH (2003) Hypolipidemic effect of an Exobiopolymer produced from a submerged mycelial culture of Hericium erinaceus. Biosci Biotechnol Biochem 67: 1292-1298. 
14. Wang XY, Yin JY, Zhao MM, Liu SY, Nie SP, et al. (2018) Gastroprotective activity of polysaccharide from Hericium erinaceus against ethanolinduced gastric mucosal lesion and pylorus ligation-induced gastric ulcer, and its antioxidant activities. Carbohydr Polym 186: 100-109.

15. Kuo HC, Lu CC, Shen CH, Tung SY, Hsieh MC, et al. (2016) Hericium erinaceus mycelium and its isolated erinacine $\mathrm{A}$ protection from MPTP-induced neurotoxicity through the ER stress, triggering an apoptosis cascade. J Transl Med 14: 78.

16. Tzeng TT, Chen CC, Chen CC, Tsay HJ, Lee LY, et al. (2018) The Cyanthin Diterpenoid and Sesterterpene Constituents of Hericium erinaceus Mycelium Ameliorate Alzheimer's Disease-Related Pathologies in APP/PS1 Transgenic Mice. Int J Mol Sci 19.

17. Tsai-Teng T, Chin-Chu C, Li-Ya L, Wan-Ping C, Chung-Kuang L, et al. (2016) Erinacine A-enriched Hericium erinaceus mycelium ameliorates Alzheimer's disease-related pathologies in APPswe/ PS1dE9 transgenic mice. J Biomed Sci 23: 49.

18. Liu PS, Chueh SH, Chen CC, Lee LY, Shiu LY (2017) Lion's Mane Medicinal Mushroom, Hericium erinaceus (Agaricomycetes), Modulates Purinoceptor-Coupled Calcium Signaling and Murine Nociceptive Behavior. Int J Med Mushrooms 19: 499-507.

19. Chu X, Zhou Y, Hu Z, Lou J, Song W, et al. (2016) 24-hour-restraint stress induces long-term depressive-like phenotypes in mice. Sci Rep 6: 32935.

20. Shah SB, Gladstone HB, Williams H, Hradek GT, Schindler RA (1995) An extended study: protective effects of nerve growth factor in neomycin-induced auditory neural degeneration. Am J Otol 16: 310 314

21. Li IC, Lee LY, Tzeng TT, Chen WP, Chen YP, et al. (2018) Neurohealth Properties of Hericium erinaceus Mycelia Enriched with Erinacines. Behav Neurol 2018: 5802634

22. Kilkenny C, Browne W, Cuthill IC, Emerson M, Altman DG, et al. (2010) Animal research: reporting in vivo experiments: the ARRIVE guidelines. Br J Pharmacol 160: 1577-1579.

23. McGrath JC, Drummond GB, McLachlan EM, Kilkenny C, Wainwright $C L$ (2010) Guidelines for reporting experiments involving animals: the ARRIVE guidelines. Br J Pharmacol 160: 1573-1576.

24. Meissner W, Prunier C, Guilloteau D, Chalon S, Gross CE, et al. (2003) Time-course of nigrostriatal degeneration in a progressive MPTPlesioned macaque model of Parkinson's disease. Mol Neurobiol 28: 209-218.

25. Shimoke K, Chiba H (2001) Nerve growth factor prevents 1-methyl4-phenyl-1,2,3,6-tetrahydropyridine-induced cell death via the Akt pathway by suppressing caspase-3-like activity using PC12 cells: relevance to therapeutical application for Parkinson's disease. J Neurosci Res 63: 402-409.

26. Presse F, Cardona B, Borsu L, Nahon JL (1997) Lithium increases melanin-concentrating hormone mRNA stability and inhibits tyrosine hydroxylase gene expression in PC12 cells. Brain Res Mol Brain Res 52: 270-283.

27. Zachor DA, Moore JF, Brezausek C, Theibert A, Percy AK (2000) Cocaine inhibits NGF-induced PC12 cells differentiation through D(1)-type dopamine receptors. Brain Res 869: 85-97.
28. Lu XL, Lin $\mathrm{YH}, \mathrm{Wu} \mathrm{Q}, \mathrm{Su} F$, Ye CH, et al. (2015) Paeonolum protects against MPP(+)-induced neurotoxicity in zebrafish and PC12 cells. BMC Complement Altern Med 15: 137.

29. Bae JW, Kim MJ, Jang CG, Lee SY (2010) Protective effects of heme oxygenase-1 against MPP(+)-induced cytotoxicity in PC-12 cells. Neurol Sci 31: 307-313.

30. Gu X, Liu L, Shen Q, Xing D (2017) Photoactivation of ERK/CREB/ VMAT2 pathway attenuates MPP(+)-induced neuronal injury in a cellular model of Parkinson's disease. Cell Signal 37: 103-114.

31. Sun Y, Selvaraj S, Pandey S, Humphrey KM, Foster JD, et al. (2018) $\mathrm{MPP}(+)$ decreases store-operated calcium entry and TRPC1 expression in Mesenchymal Stem Cell derived dopaminergic neurons. Sci Rep 8: 11715.

32. Park YS, Lee HS, Won MH, Lee JH, Lee SY, et al. (2002) Effect of an exo-polysaccharide from the culture broth of Hericium erinaceus on enhancement of growth and differentiation of rat adrenal nerve cells. Cytotechnology 39: 155-162.

33. Howells DW, Porritt MJ, Wong JY, Batchelor PE, Kalnins R, et al. (2000) Reduced BDNF mRNA expression in the Parkinson's disease substantia nigra. Exp Neurol 166: 127-135.

34. Lorigados Pedre L, Pavon Fuentes N, Alvarez Gonzalez L, McRae A, Serrano Sanchez T, et al. (2002) Nerve growth factor levels in Parkinson disease and experimental parkinsonian rats. Brain Res 952: 122-127.

35. Cheung WM, Hui WS, Chu PW, Chiu SW, Ip NY (2000) Ganoderma extract activates MAP kinases and induces the neuronal differentiation of rat pheochromocytoma PC12 cells. FEBS Lett 486: 291-296.

36. Lee IK, Kim YS, Jang YW, Jung JY, Yun BS (2007) New antioxidant polyphenols from the medicinal mushroom Inonotus obliquus. Bioorg Med Chem Lett 17: 6678-6681.

37. Oropeza-Guerrero MP, Santos-Sanchez NF, Salas-Coronado $R$, Valadez-Blanco R, Hernandez-Carlos B, et al. (2018) Productivity and Antioxidant Activity of Wild, Reconstituted, and Hybrid Strains of the Pink Oyster Mushroom, Pleurotus djamor (Agaricomycetes), from Mexico. Int J Med Mushrooms 20: 607-621.

38. Kalia LV, Lang AE (2015) Parkinson's disease. Lancet 386: 896-912.

39. Reksidler AB, Lima MM, Dombrowski P, Andersen ML, Zanata SM, et al. (2008) Repeated intranigral MPTP administration: a new protocol of prolonged locomotor impairment mimicking Parkinson's disease. J Neurosci Methods 167: 268-277.

40. Fitzpatrick PF (1991) Steady-state kinetic mechanism of rat tyrosine hydroxylase. Biochemistry 30: 3658-3662

41. Kaushik P, Gorin F, Vali S (2007) Dynamics of tyrosine hydroxylase mediated regulation of dopamine synthesis. J Comput Neurosci 22 147-160.

42. Bjugstad KB, Redmond DE Jr, Teng YD, Elsworth JD, Roth RH, et al. (2005) Neural stem cells implanted into MPTP-treated monkeys increase the size of endogenous tyrosine hydroxylase-positive cells found in the striatum: a return to control measures. Cell Transplant 14: 183-192. 